\title{
Direct Fabrication of Functional Ultrathin Single-crystal Nanowires from Quasi-one- dimensional Van Der Waals Crystals
}

Xue Liu,${ }^{\dagger}$ Jinyu Liu, ${ }^{\dagger}$ Liubov Yu. Antipina, $,{ }^{\ddagger},, l$ Jin Hu,${ }^{\dagger}$ Chunlei Yue, ${ }^{\dagger}$ Ana M. Sanchez, ${ }^{\perp}$ Pavel B. Sorokin, ${ }^{\ddagger}, l$ Zhiqiang Mao, ${ }^{\dagger}$ Jiang Wei ${ }^{*} \dagger$

${ }^{\dagger}$ Department of Physics and Engineering Physics, Tulane University, New Orleans, Louisiana 70118, USA

Technological Institute of Superhard and Novel Carbon Materials, Moscow, 142190, Russian Federation

${ }^{\S}$ Moscow Institute of Physics and Technology, Dolgoprudny, 141700, Russian Federation

"National University of Science and Technology MISiS, Moscow, 119049, Russian Federation

${ }^{\perp}$ Department of Physics, University of Warwick, Coventry, CV4 7AL, UK

\section{Supplementary Information}

\section{Method of bulk TPdS crystal growth}

Single crystals of TPdS were grown using chemical vapor transport. The mixture of thoroughly ground stoichiometric $\mathrm{Ta}, \mathrm{Pd}$ and Se element powders was loaded into a quartz tube and sealed under vacuum. The tube with loaded materials was pre-heated for a week at $750{ }^{\circ} \mathrm{C}$. The obtained powder was then ground again and transferred to a new quartz tube (inside diameter $=0.9 \mathrm{~cm}$ ) together with $75 \mathrm{mg}$ iodine as transport agent. The tube was vacuumed and sealed at the point $15 \mathrm{~cm}$ away from charge end and then put into a double zone furnace. The charge end and the other end were heated to $850{ }^{\circ} \mathrm{C}$ and $900{ }^{\circ} \mathrm{C}$ respectively. After holding for one day, the temperature was reversed with charge end at $900{ }^{\circ} \mathrm{C}$ and cold end at $850{ }^{\circ} \mathrm{C}$. The furnace was shut down after one week. Long needle-like crystals (up to a few centimeters) with silver luster formed at the cold end. Preliminary composition analyses on different crystals using Energy Dispersive X-ray Spectrometer showed the ratio of Ta, $\mathrm{Pd}$, and $\mathrm{Se}$ atoms very close to 2:3:8 as shown by figure S1. The powder X-ray diffraction as indicated by figure S2 at room temperature confirmed the phase to be TPdS ${ }^{1}$. All the diffraction peaks can be indexed according to the known structure of TPdS, although there is difference of peak intensity and some missing peaks caused by strongly preferred orientation issue. 

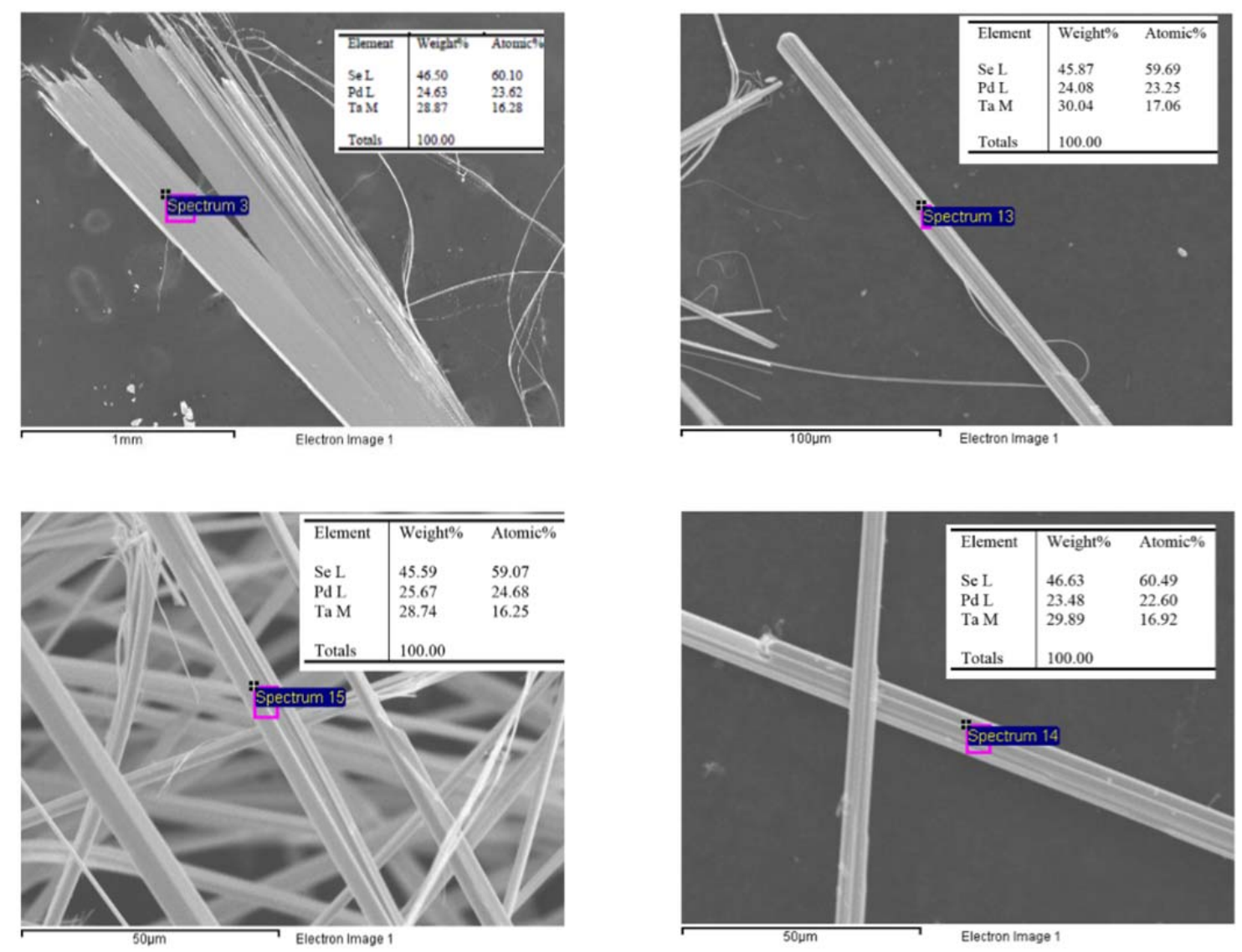

Figure S1. Energy Dispersive X-ray Spectroscopy data of the TPdS bulk crystal on multiple spots at different locations of the sample. 


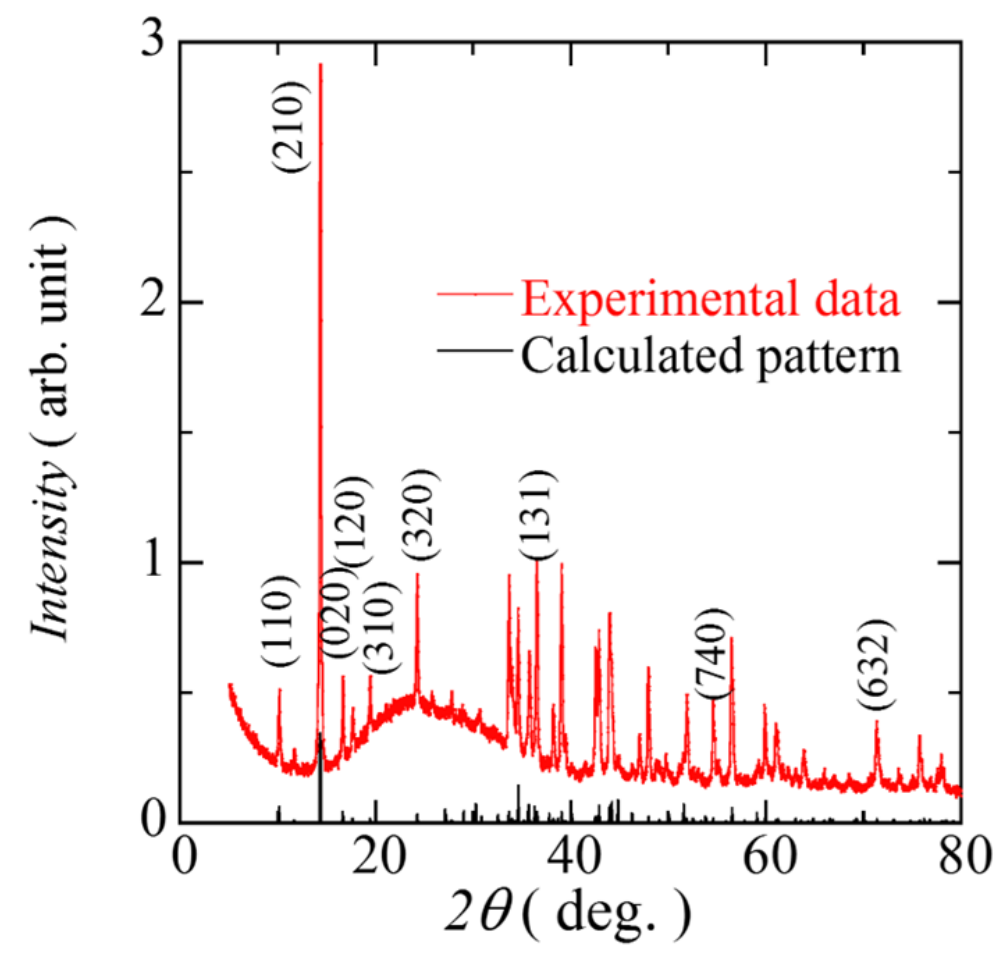

Figure S2. Powder X-Ray diffraction pattern (red colored plot) of pulverized TPdS crystals at room temperature and calculated diffraction pattern (black colored plot).

\section{Temperature dependent resistivity measurement for bulk TPdS crystal}

Bulk crystal's resistivity was measured using four-probe method in Physical Property Measurement System (PPMS, Quantum Design. Inc).

The temperature dependence of resistivity exhibits a semiconducting behavior as plotted in figure S3. A simple fit of the resistivity data to the Arrhenius equation $\ln \rho=\ln \rho_{0}+$ $E_{g} /\left(2 k_{B} T\right)$ within the temperature range of $250-300 \mathrm{~K}$ yields an activation energy of $35 \mathrm{meV}$, which is much smaller than the expected band gap $(\sim 0.5 \mathrm{eV})$ from the band structure calculation. 


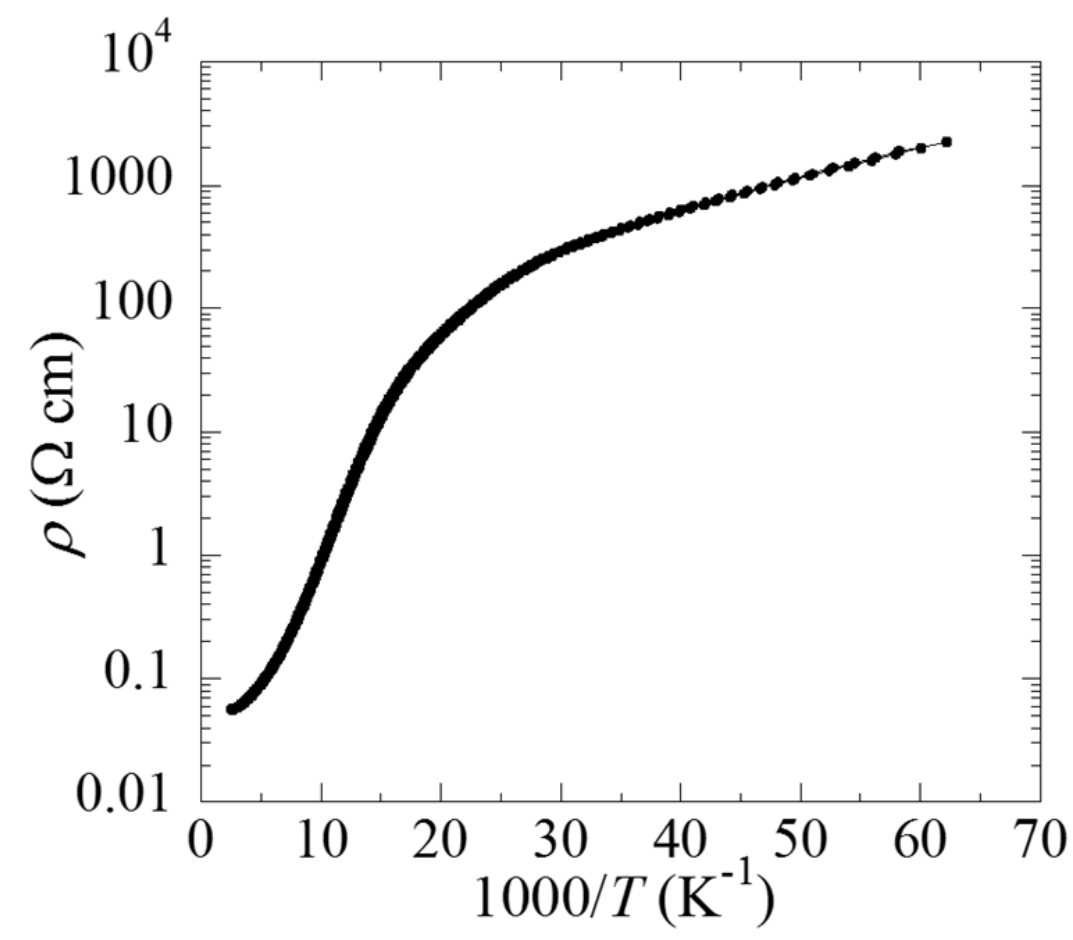

Figure S3. Temperature dependent resistivity along needle direction for bulk crystal. $\rho$ (300 $\mathrm{K})=0.054 \Omega \cdot \mathrm{cm}$ and $\rho(16 \mathrm{~K})=2250 \Omega \cdot \mathrm{cm}$.

\section{Atomic and electronic structure calculation of TPdS crystal}

All calculations of atomic and electronic structure of TPdS structures were performed by DFT theory ${ }^{2,3}$ within the PBE-PAW approximation ${ }^{4}$ with the periodic boundary conditions using a Vienna Ab-initio Simulation Package. ${ }^{5-7}$ The plane-wave energy cutoff was equal to $325 \mathrm{eV}$. To calculate the equilibrium atomic structures, the Brillouin zone was sampled according to the Monkhorst-Pack ${ }^{8}$ scheme with a grid $4 \times 6 \times 10$ k-point for bulk and $1 \times 1 \times 10 \mathrm{k}$-point for $1 \mathrm{D}$ structures. To avoid the interaction between the neighboring nanowires images, the vacuum space between them was greater than $15 \AA$ in all non-periodic direction. The structural relaxation was performed until the forces acting on each atom were less than $0.02 \mathrm{eV} / \AA$. The structural parameters of the TPdS system were calculated within error of less than $3 \%$ (compared with the experimental data taken from Ref. ${ }^{1,9}: a_{\text {calc }}=15.53 \AA, b_{\text {calc }}=10.95 \AA c_{\text {calc }}=3.61 \AA$ and $a_{\text {exp }}=15.15 \AA, b_{\text {exp }}=10.63 \AA c_{\text {exp }}=3.54 \AA$ ).

\section{Optical identification of exfoliated TPdS nanowires}

The exfoliated TPdS nanowires on Si substrate ( $285 \mathrm{~nm} \mathrm{SiO}_{2}$ on top) appear to have different colors corresponding to different thicknesses, similar to the case of exfoliated 2D vdW materials on Si substrate. As indicated by figure S4, using bright field mode with 100X magnification under optical microscope, the thicker TPdS nanowires $(>100 \mathrm{~nm})$ display light green color with clear edge distinguishable from the substrate. However, the color of thinner nanowires ( 80 
$\mathrm{nm} \sim 15 \mathrm{~nm}$ ) becomes dark blue with decreasing brightness and contrast. Extremely thin nanowire down to $5 \mathrm{~nm}$ is still visible.
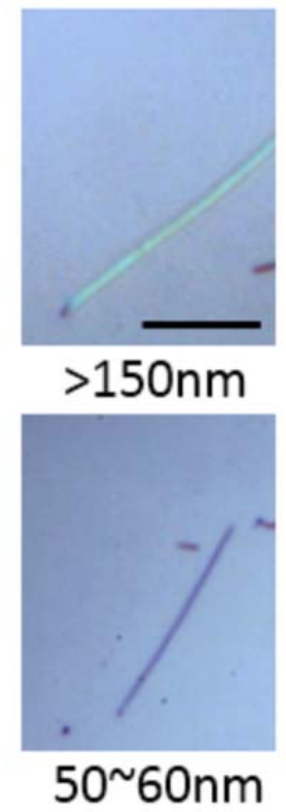

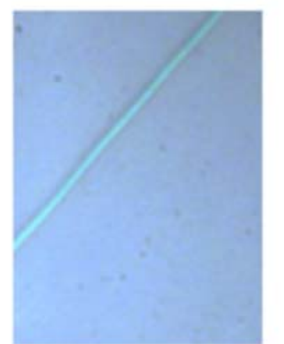

$100 \mathrm{~nm}$

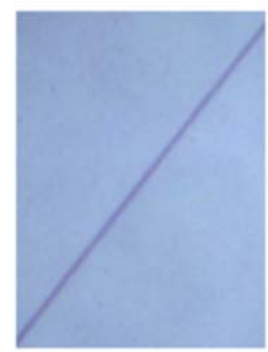

$30 \mathrm{~nm}$

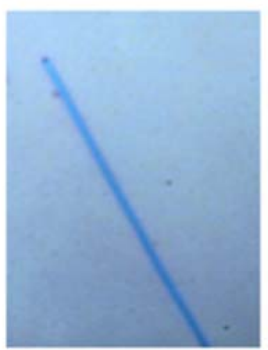

$80 \mathrm{~nm}$

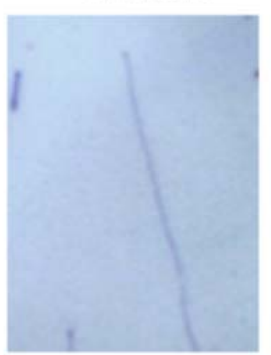

$15^{\sim} 20 \mathrm{~nm}$
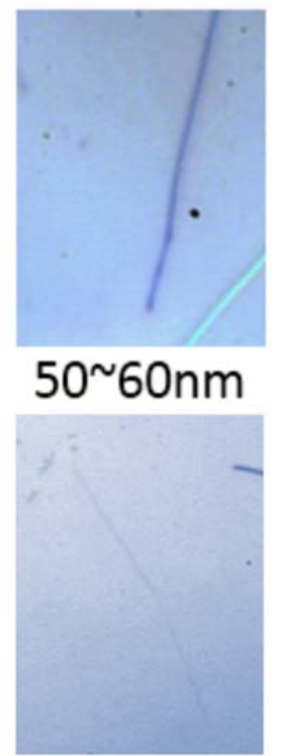

$5 \mathrm{~nm}$

Figure S4. Color mapping of exfoliated TPdS nanowires with different thicknesses $(150 \mathrm{~nm}$ to $5 \mathrm{~nm})$. Scale bar is $5 \mu \mathrm{m}$.

\section{Atomic resolution STEM analysis of TPdS nanowires}

Atomic resolution ADF-STEM images of TPdS nanowires were obtained using a JEOL annular field detector with a fine-imaging probe, at a probe current of approximately $23 \mathrm{pA}$ with a convergence semi-angle of $22 \mathrm{mrad}$ and an inner semi-angle of 45-50 mrad. The TPdS samples were prepared by depositing exfoliated TPdS nanowires on both graphene and carbon film supported TEM grids. Images of two different projections of the nanowires thicker than $50 \mathrm{~nm}$ can be observed in figure S5. TPdS structure of these nanowires were determined by comparing ADF-STEM images along a-axis (figure S5a) and b-axis (figure S5b) with the simulated atomic structure images. 
(a)

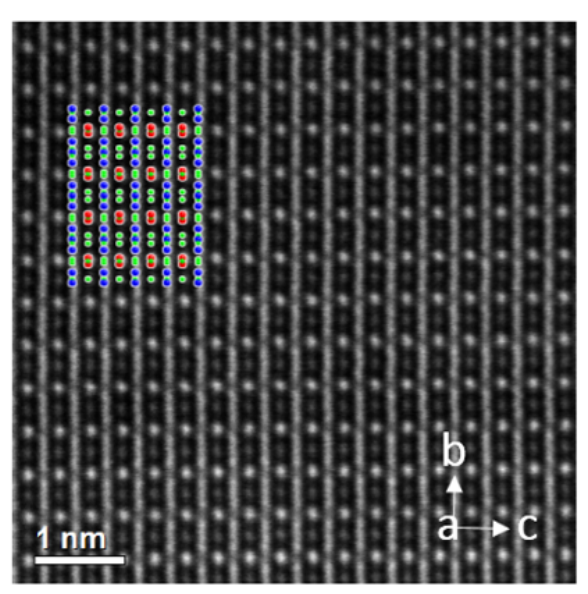

(b)

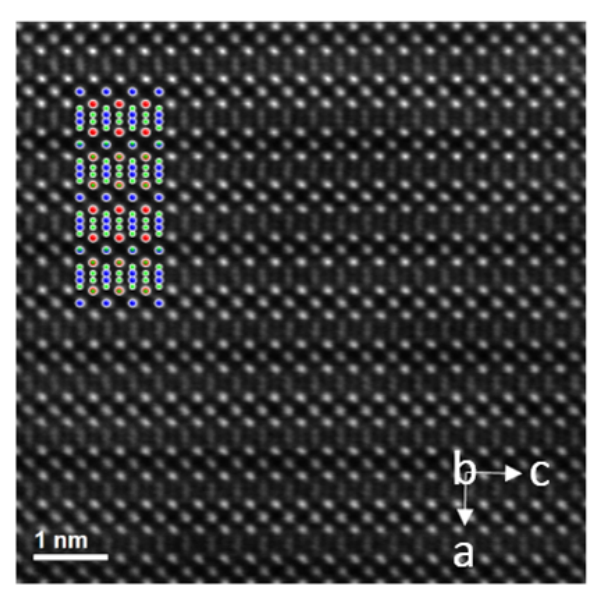

Figure S5. ADF-STEM images of TPdS nanowires overlaid by the images of simulated crystal structure (shown as the insets). The viewing directions are along (a) a-axis and (b) b-axis respectively.

EDX analysis were performed with probe currents of approximately $200 \mathrm{pA}$ with a convergence semi-angle of $28 \mathrm{mrad}$ and collected with an Oxford Instruments X-Max Windowless Detector with an area of $100 \mathrm{~mm}^{2}$. Figure S6 shows the atomic distribution of Ta, $\mathrm{Pd}$ and Se elements in the TPdS structure observed along b-axis.

(a)

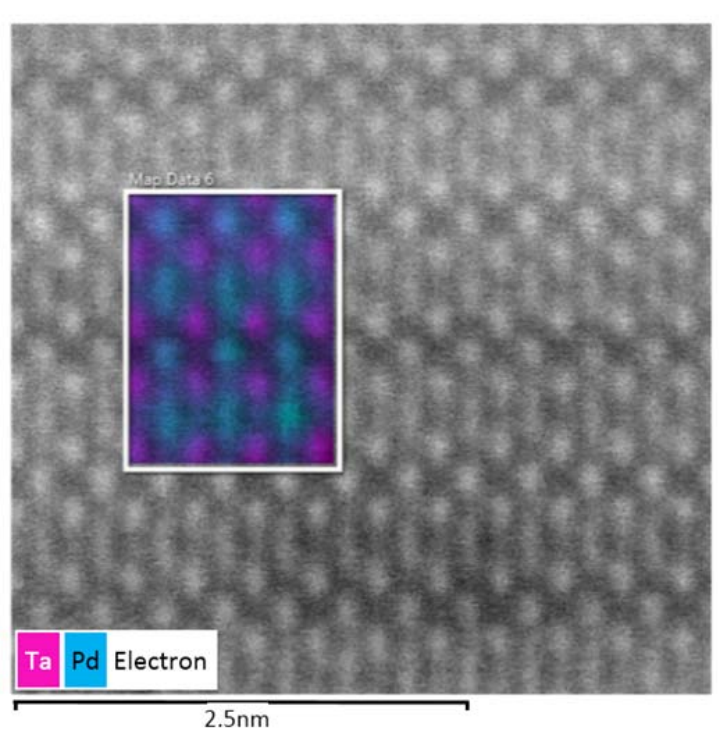

(b)
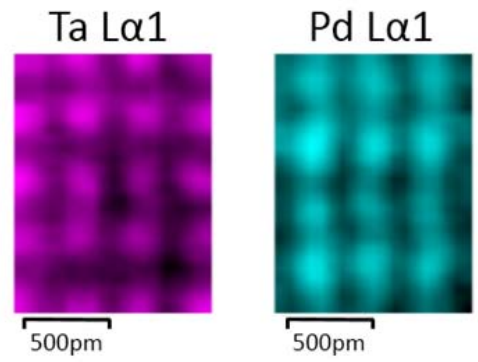

Se K $\alpha 1$

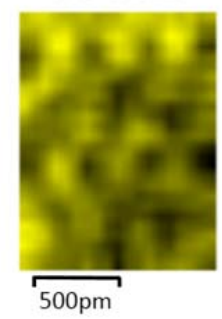

Figure S6. (a) Atomic resolution EDX of TPdS structure along the b-axis. Inset shows the transition metal (Ta and Pd) colored map matching with the crystal structure. (b) Lateral distribution of Ta (magenta), Pd (cyan) and Se (yellow) elements. 


\section{Wulff construction}

The nanowire's equilibrium shape was estimated by Wulff construction approach. ${ }^{10}$ In this method the optimal crystal shape is determined by the solution to the energy minimization problem of $\sum_{j} \gamma_{j} S_{j}$, where $\gamma_{j}$ and $S_{j}$ are energy and area of $j$ surface.

We calculated the energy of TPdS crystal surface (001), (010), (100), (110), (120) and (210) using the equation

$$
\gamma_{i}=\frac{1}{2 S}\left(E_{i}(N)-N E_{\text {bulk }}\right),
$$

where $E_{i}(N)$ is the energy of N-layer slab with corresponding surface orientation with the surface area $S, E_{\text {bulk }}$ is bulk energy per formula unit $\mathrm{Ta}_{2} \mathrm{Pd}_{3} \mathrm{Se}_{8}$. The factor of $1 / 2$ accounts for the two surfaces of the slab. The eq. (1) was used for the evaluation of the slabs surface energy in the range of the thickness from 2 to 25 formula units.

The constructed equilibrium shape of the nanowire contains four preferred facets ((100), (010), (110), (210)), see
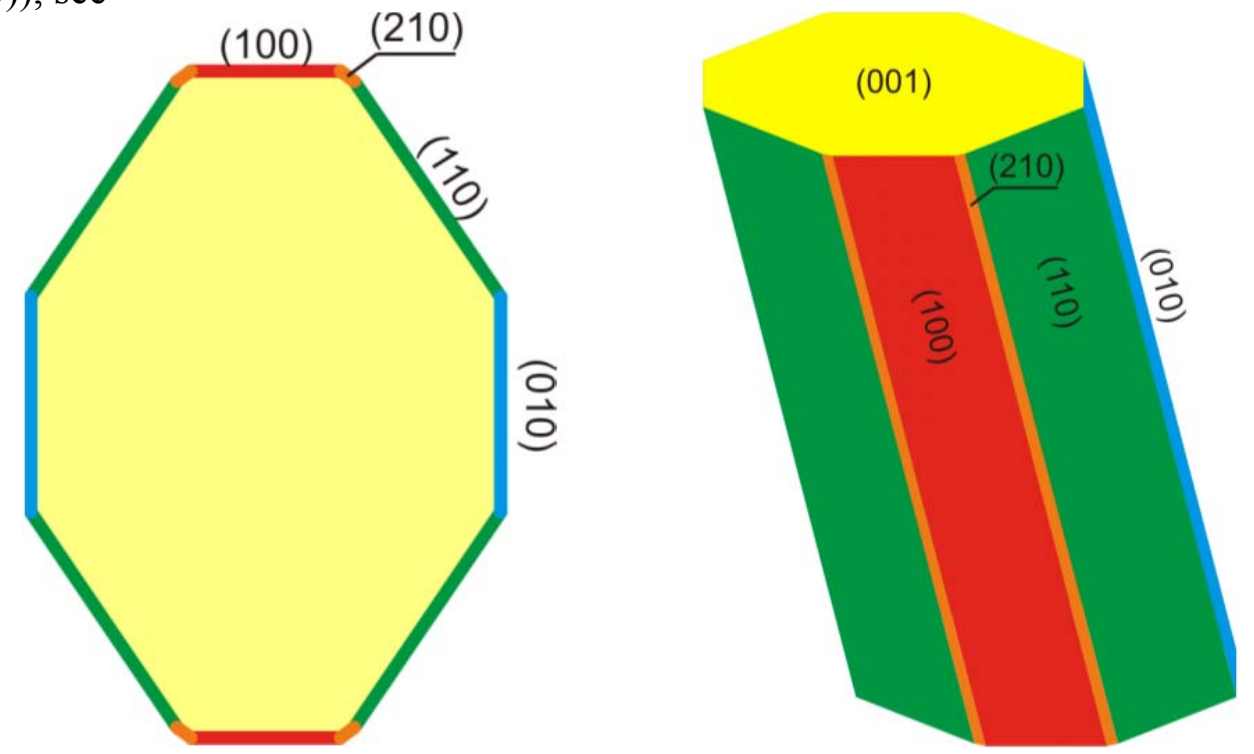

Figure S7. Cross section and perspective views of the simulated equilibrium shape of TPdS nanowire constructed with four preferred facets ((100), (010), (110), (210)).

\section{Transport data analysis}

We investigated more than 100 FET devices based on TPdS nanowires with thicknesses spanning from $5 \mathrm{~nm}$ to $65 \mathrm{~nm}$. The thickness dependence of the extracted field effect mobility is plotted in figure S8. It can be seen that TPdS nanowires with a thickness $12 \mathrm{~nm}$ to $30 \mathrm{~nm}$ have relatively high mobility. 


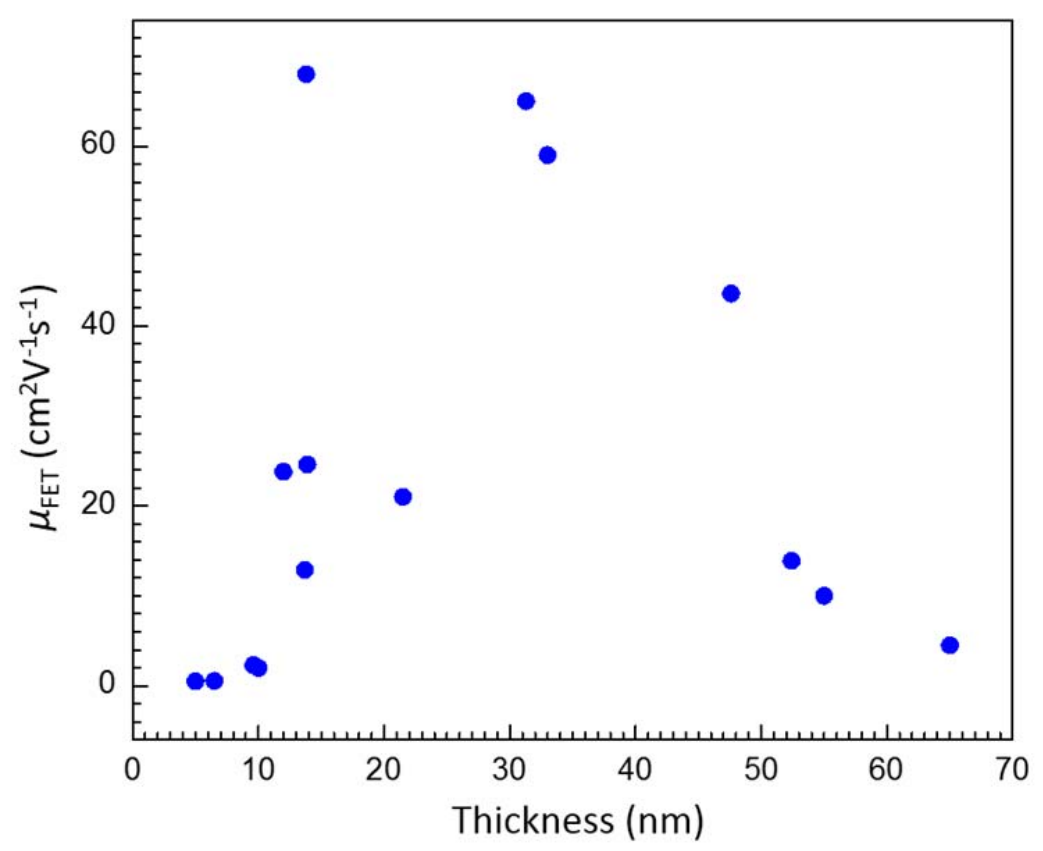

Figure S8. Thickness dependence of field effect mobility extracted from 15 TPdS nanowire FETs.

TPdS nanowire device's switching behavior can be explained based on the band diagram shown in figure S9. The main charge carrier (electron) at room temperature is mainly originated from the defects in TPdS nanowire through thermal activation. Therefore, the crystal appears as $\mathrm{n}$ doped semiconductor. At room temperature, all our devices show linear I-V, from which we conclude that Schottky barrier between Pd contact and TPdS nanowire is very small $(<26 \mathrm{meV}$ at least). However, due to the semiconducting properties of TPdS, electrostatically induced band bending via gating is expected to occur in our devices as illustrated in figure S9a-c. In order to accurately locate the energy level of defects, back gate voltage is increased from 0 to $80 \mathrm{~V}$, and for each value we extract the thermal activation energy $E_{\mathrm{a}}$ from the temperature dependence data by performing Arrhenius fitting as plotted in figure S9d. There is a clear turning point where $E_{\mathrm{a}}$ vs. $V_{\mathrm{bg}}$ changes its curvature. Such point corresponds to the so-called flat band condition, i.e. the band between source drain electrodes becomes flat as illustrated in figure S9b. The thermal activation energy extracted at flat band condition $\left(V_{\mathrm{bg}}=V_{\mathrm{FB}}\right)$ reflects the intrinsic energy difference between the defect level and the edge of conduction band. 

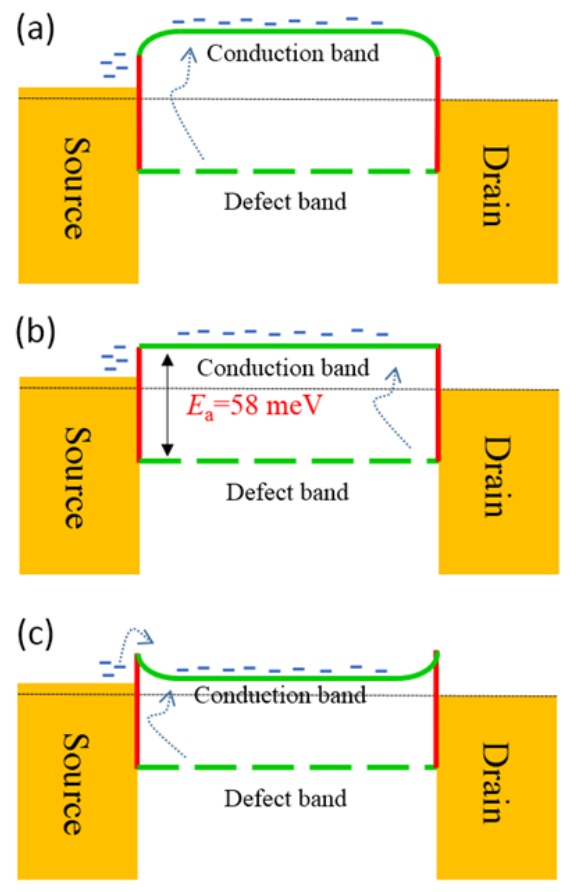

$E_{\mathrm{f}}$

$V_{\mathrm{bg}}=0 \mathrm{~V}$

(d)

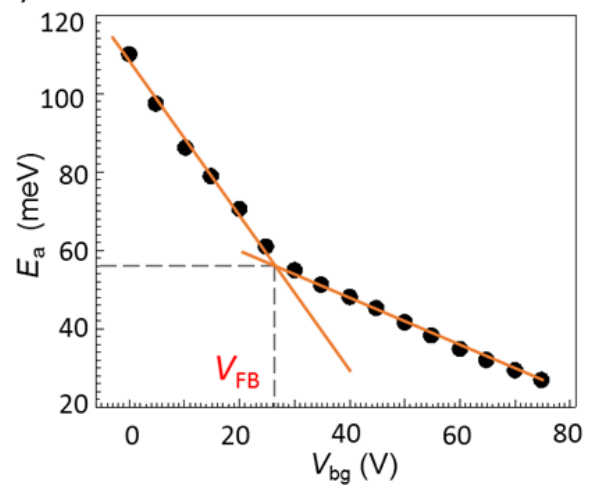

$V_{\mathrm{bg}}>V_{\mathrm{FB}}$

Figure S9. Energy band diagrams of TPdS FET device with gate voltage set to bend the band (a) upward, (b) flat, and (c) downward. (d) Extracted thermal activation energy $E_{\mathrm{a}}$ at back gate voltage $V_{\mathrm{bg}}$ set at different values. $V_{\mathrm{FB}}$ is the corresponding back gate voltage when the band is bended flat as illustrated in (b).

In low temperature $(<140 \mathrm{~K})$ region, the temperature dependence of the source drain current follows the relation $\ln I \propto T^{-0.5}$, which indicates a variable range hopping (VRH) behavior. ${ }^{11}$ However, both 1D Mott's VRH and Efros and Shklovskii variable range hopping (ES-VRH) ${ }^{12}$ give the same temperature dependence.

To further determine the dominating mechanism, the differential conductance was taken from $\mathrm{I}-\mathrm{V}$ curves at different temperatures for a few devices as shown in figure S10. It can be seen that a zero-bias anomaly (sharp dip at low bias) emerges below $180 \mathrm{~K}$, which is a clear signature of electron-electron interaction ${ }^{13-15}$ in accordance with the gradual opening of a Coulomb gap at low temperature. In this case, the coulomb interaction plays an important role at low temperature, for which ES-VRH model should be used to fit our data by using the expression $I(T)=A_{0}^{\prime} e^{\frac{-\sqrt{T^{*}}}{\sqrt{T}}}$, where $A_{0}^{\prime}$ and $T^{*}$ are fitting parameters and $T^{*}=\frac{2.8 e^{2}}{4 \pi \varepsilon \varepsilon_{0} a k_{B}}$, which is the characteristic ES temperature. Since the dielectric constant of our TPdS nanowire is still unavailable from literatures, we used the typical value for silicon nanowire $\varepsilon=11.2 .^{16,17}$ and estimated the localization length $a$ to be $\sim 9.64 \mathrm{~nm}$, which is a comparable value for disordered $1 \mathrm{D}$ nanowire system, such as carbon nanotubes. ${ }^{18}$ 

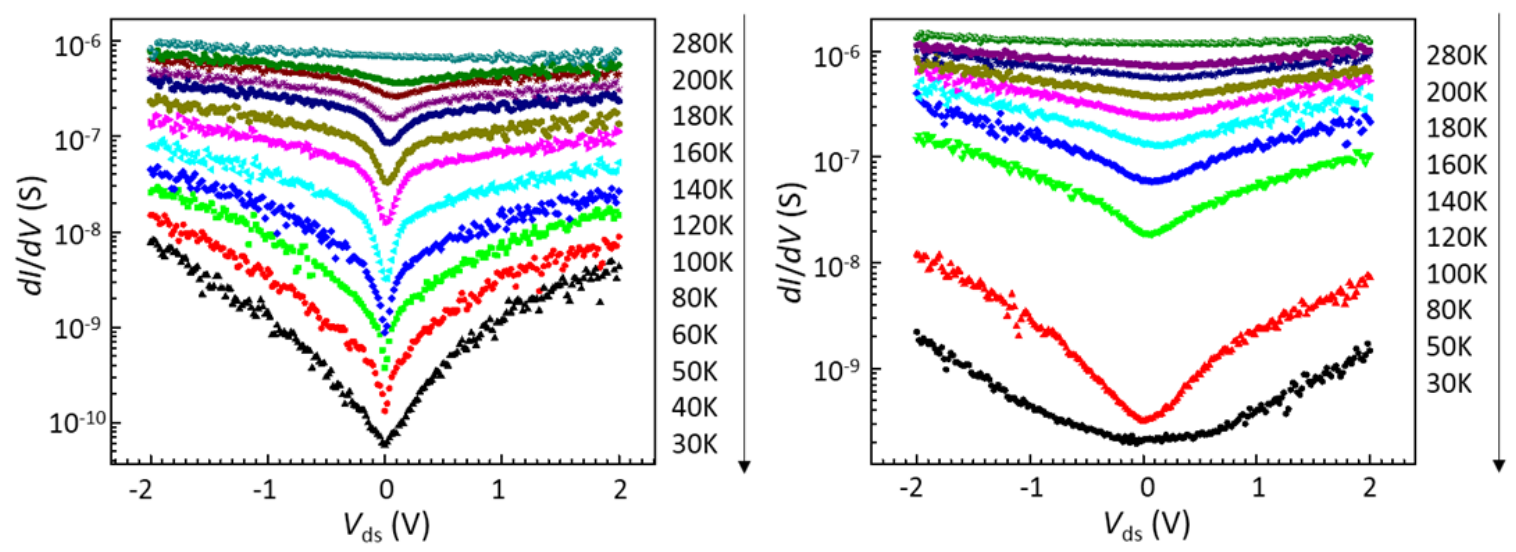

Figure S10. Differential conductance derived from I-V curves at different temperature ranging from $30 \mathrm{~K}$ to $280 \mathrm{~K}$ based on two different devices (the left one is a device with $28 \mathrm{~nm}$ nanowire and the right one is a device with $47.6 \mathrm{~nm}$ nanowire). At low temperature a zero-bias anomaly (sharp dip) emerges which is not apparent at temperatures above $180 \mathrm{~K}$.

\section{TPdS device environmental stability tested with transport characterization}

TPdS FET devices (with a $14.5 \mathrm{~nm}$ nanowire) were stored in ambient environment and tested for their stability by measuring the transconductance sequentially for 40 days. As shown in figure S11, the transconductance shows little change over 40 days. We focused on nanowires with thicknesses in the range of $12 \mathrm{~nm}$ to $30 \mathrm{~nm}$, which shows the best performance as discussed in the mobility histogram (figure S8). For thinner nanowires (less than $10 \mathrm{~nm}$ ), contact resistance between contact metal (we use gold as contact metal) and nanowire becomes dominant, which makes transport measurement on thinner nanowires unsuitable for stability test. 


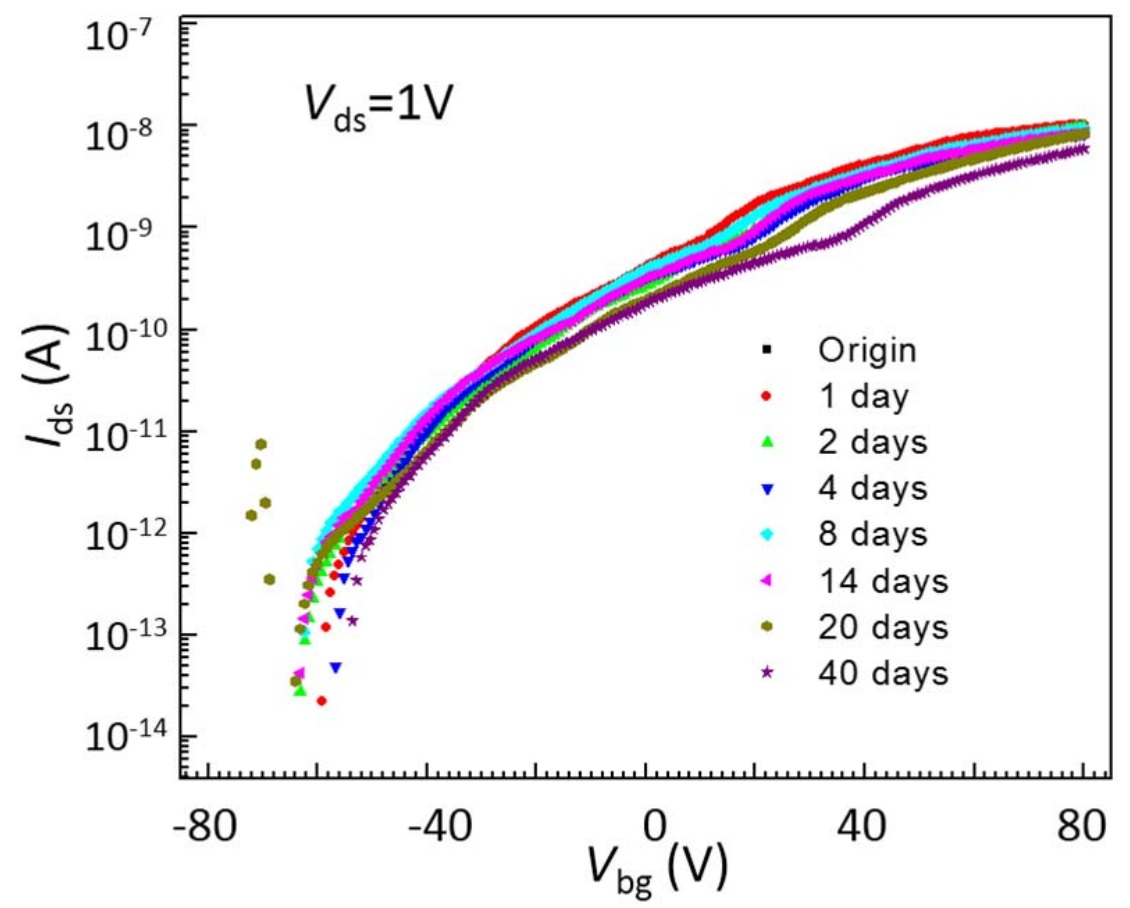

Figure S11. Transconductance data of an exfoliated TPdS nanowire device measured at different time point in a period of 40 days in ambient environment.

\section{References:}

$1 \quad$ Keszler, D. A., Ibers, J. A., Maoyu, S. \& Jiaxi, L. New ternary and quaternary transitionmetal selenides: Syntheses and characterization. Journal of Solid State Chemistry 57, 6881 (1985).

2 Hohenberg, P. \& Kohn, W. Inhomogeneous Electron Gas. Physical Review 136, B864B871 (1964).

3 Kohn, W. \& Sham, L. J. Self-consistent equations including exchange and correlation effects. Physical review 140, A1133 (1965).

4 Perdew, J. P., Burke, K. \& Ernzerhof, M. Generalized gradient approximation made simple. Physical review letters 77, 3865 (1996).

$5 \quad$ Kresse, G. \& Hafner, J. Ab initio molecular dynamics for liquid metals. Physical Review $B$ 47, 558 (1993).

6 Kresse, G. \& Hafner, J. Ab initio molecular-dynamics simulation of the liquid-metalamorphous-semiconductor transition in germanium. Physical Review B 49, 14251 (1994).

7 Kresse, G. \& Furthmüller, J. Efficient iterative schemes for ab initio total-energy calculations using a plane-wave basis set. Physical Review B 54, 11169 (1996).

8 Monkhorst, H. J. \& Pack, J. D. Special points for Brillouin-zone integrations. Physical Review B 13, 5188 (1976). 
9 Keszler, D. A. \& Ibers, J. A. A new structural type in ternary chalcogenide chemistry: Structure and properties of Nb2Pd3Se8. Journal of Solid State Chemistry 52, 73-79 (1984).

10 Wul, G. Zur Frage der Geschwindigkeit des Wachstums und der Auflosung der Kristall achen. Z. Kristallogr 34, 449-530 (1901).

11 Mott, N. F. \& Davis, E. A. Electronic Processes in Non-Crystalline Materials. (OuP Oxford, 2012).

12 Shklovskii, B. I. \& Efros, A. L. Electronic properties of doped semiconductors. Vol. 45 (Springer Science \& Business Media, 2013).

13 Aharony, A., Zhang, Y. \& Sarachik, M. P. Universal crossover in variable range hopping with Coulomb interactions. Physical Review Letters 68, 3900-3903 (1992).

14 Sandow, B., Gloos, K., Rentzsch, R., Ionov, A. N. \& Schirmacher, W. Electronic Correlation Effects and the Coulomb Gap at Finite Temperature. Physical Review Letters 86, 18451848 (2001).

15 Butko, V. Y., DiTusa, J. F. \& Adams, P. W. Coulomb Gap: How a Metal Film Becomes an Insulator. Physical Review Letters 84, 1543-1546 (2000).

16 Arbiol, J. \& Xiong, Q. Semiconductor Nanowires: Materials, Synthesis, Characterization and Applications. (Elsevier Science, 2015).

17 Blatt, F. J. Modern Physics. (McGraw-Hill, 1992).

18 Wang, D. P. et al. Hopping conduction in disordered carbon nanotubes. Solid State Communications 142, 287-291 (2007). 